\title{
Call for Papers: Issue 1/2017
}

\section{Human Information Behavior in IS Development and Use}

\author{
Glenn J. Browne - Christy M. K. Cheung • \\ Armin Heinzl • René Riedl
}

Published online: 9 June 2015

(C) Springer Fachmedien Wiesbaden 2015

\section{Special Issue}

Human information behavior (HIB) is a multi-disciplinary field at the intersection of different scientific disciplines, including information systems (IS), psychology, information science, sociology, and neuroscience. It is concerned with explaining and predicting human behaviors towards information. This includes behaviors relating to acquiring, interpreting, using, and sharing information, in addition to behaviors relating to limiting, filtering, and stopping the information intake. Information pathologies are of special relevance to HIB research. They describe misguided and malicious behaviors that cause relevant information not to be produced, absorbed, used, or shared. Examples are information avoidance, information hiding, and the mali-

G. J. Browne

Rawls College of Business, Texas Tech University, Lubbock, USA

e-mail: glenn.browne@ttu.edu

\section{M. K. Cheung}

Department of Finance and Decision Sciences, School of Business, Hong Kong Baptist University, Hong Kong, China e-mail: ccheung@hkbu.edu.hk

\section{A. Heinzl ( $ه)$}

Chair in General Management and Information Systems, Mannheim University, Mannheim, Germany

e-mail: heinzl@uni-mannheim.de

R. Riedl

University of Applied Sciences Upper Austria, Linz, Austria e-mail: rene.riedl@fh-steyr.at

\section{R. Riedl}

University of Linz, Linz, Austria cious manipulation of information. HIB topics bring together researchers with diverse backgrounds, epistemological stances, and research methods. The unit of observation is the individual, the group, the organization, and the society as a whole.

This special issue seeks contributions that explicitly address the cognitive and affective processes that relate to information behaviors and the types of information behaviors evident in the IS literature. Furthermore, it seeks contributions dealing with the information construct itself, in addition to issues relating to information quality, information relevance, and information value.

This BISE journal special issue is open to contributions regardless of the scientific methodology chosen. Innovative approaches such as the use of neurophysiology (NeuroIS) are highly appreciated; but the special issue is not restricted to these methods. The special issue welcomes IS research contributions which explicitly deal with HIB aspects related to the development and use of IS.

Contributions from research and business practice on the following (and related) topics are invited:

- Theories and models which address specific phenomena of HIB

- Information behaviors such as information searching, stopping or sharing

- Information pathologies such as avoidance or hiding

- Research methodologies that specifically apply for HIB

- Cultural factors in HIB

- Individual factors in HIB, such as age, gender, or personality

- Design science approaches to HIB topics

- Case studies relating to HIB in IS

- Studies on digital literacy and digital inclusion if they are related to HIB themes 


\section{Submission Guidelines}

Please submit papers for the sections Research Paper and State of the Art by 1 March 2016 at the latest via the journal's online submission system (http://www.editorialmanager.com/buis/). Please observe the instructions regarding the format and size of contributions to Business \& Information Systems Engineering (BISE). Papers should not exceed 10 pages; this amounts to 50,000 characters including spaces, minus 5000 characters per page for illustrations. Detailed authors' guidelines can be downloaded from http://www.bise-journal.org.

All papers will be reviewed anonymously (double-blind process) by at least two referees with regard to relevance, originality, and research quality. In addition to the editors of the journal, including those of this special issue, distinguished international professionals with scientific and practical backgrounds will be involved in the review process.

\section{Schedule}

Submission deadline: 1 March 2016

Author notification: 26 April 2016

Completion of first revision: 28 June 2016

Author notification: 16 August 2016

Completion of second revision: 20 September 2016

Editorial deadline: 15 October 2016

Planned publication date: February 2017 